\title{
Endoscopic Endonasal Odontoidectomy Preserving Atlantoaxial Stability: a Pediatric Case
}

\author{
Pierlorenzo Veiceschi ${ }^{1}$ Fabio Pozzi ${ }^{1}$ Francesco Restelli ${ }^{1}$ Tommaso Alfiero ${ }^{1}$ Paolo Castelnuovo ${ }^{2}$ \\ Davide Locatelli ${ }^{3}$
}

${ }^{1}$ Department of Neurosurgery, Ospedale di Circolo Fondazione Macchi, Varese, Italy

${ }^{2}$ Department of Otorhinolaryngology, University of Insubria, Ospedale di Circolo Fondazione Macchi, Varese, Italy

${ }^{3}$ Department of Neurosurgery, University of Insubria, Ospedale di Circolo Fondazione Macchi, Varese, Italy

J Neurol Surg B 2021;82(suppl S1):S2-S3.
Address for correspondence Pierlorenzo Veiceschi, MD, Department of Neurosurgery, Ospedale di Circolo Fondazione Macchi, Via Guicciardini 9, 21100 Varese, Italy (e-mail: pierloveiceschi@gmail.com).

\begin{abstract}
Keywords

- craniocervical junction

- odontoidectomy

- basilar invagination

- Chiari I

- endoscopic skull base

Objectives We illustrate endoscopic endonasal odontoidectomy for the Chiari-I malformation respecting craniovertebral junction (CVJ) stability.

Design Case report of a 12-year-old girl affected by the Chiari-I malformation. Magnetic resonance imaging (MRI) showed tonsillar herniation, basilar invagination, and dental retroversion, causing angulation and compression of the bulbomedullary junction. Patient underwent endoscopic third ventriculostomy (ETV) with reduction of ventricular size and resolution of gait disturbances, but she complained the Valsalvainduced headaches, hiccup, and dysesthesias in the lower limbs. Endoscopic endonasal odontoidectomy was chosen to decompress the cervicomedullary junction.

Setting The research was conducted at University Hospital "Ospedale di Circolo," Department of Neurosurgery at Varese in Italy.

Participants Patients were from neurosurgical and ENT (ear, nose, and throat) skull base team.

Main Outcome Measures A bilateral paraseptal approach was performed, using a four-hand technique. After resection of posterior edge of the nasal septum, the choana is entered and a rhinopharynx muscle-mucosal flap is dissected subperiosteal and transposed in oral cavity. The CV] is exposed and, using neuronavigation and neuromonitoring, odontoidectomy is fulfilled until dura is reached, preserving the anterior arch of $\mathrm{C} 1$. Reconstruction is obtained suturing the flap previously harvested.
\end{abstract}

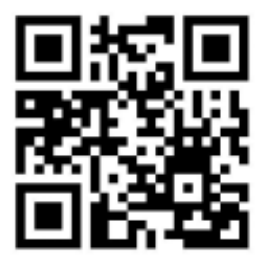

received

March 1, 2019 accepted after revision September 29, 2019 published online March 4, 2020

\section{Conflict of Interest \\ None declared.}

www.thieme.com/skullbasevideos

www.thieme.com/jnlsbvideos

DOI https://doi.org/ $10.1055 / \mathrm{s}-0039-3402797$. ISSN 2193-6331.

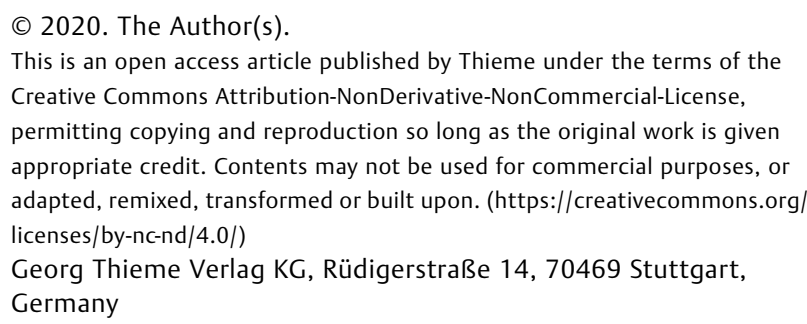


Results Postoperative course was unremarkable and the patient experienced improvement of symptoms. Postoperative MRI documented the appearance of tight cerebrospinal fluid (CSF) film anterior to bulbomedullary junction and in retrotonsillar spaces, opening of the bulbomedullary angle, and slight tonsils reduction. No CV] instability was occurred with any need of posterior fixation.

Conclusion Endoscopic endonasal odontoidectomy is a feasible approach for CV] malformation. In this case, bulbar decompression was achieved preserving CV] stability and avoiding posterior fixation.

The link to the video can be found at: https://youtu.be/VlobocHfCuc.

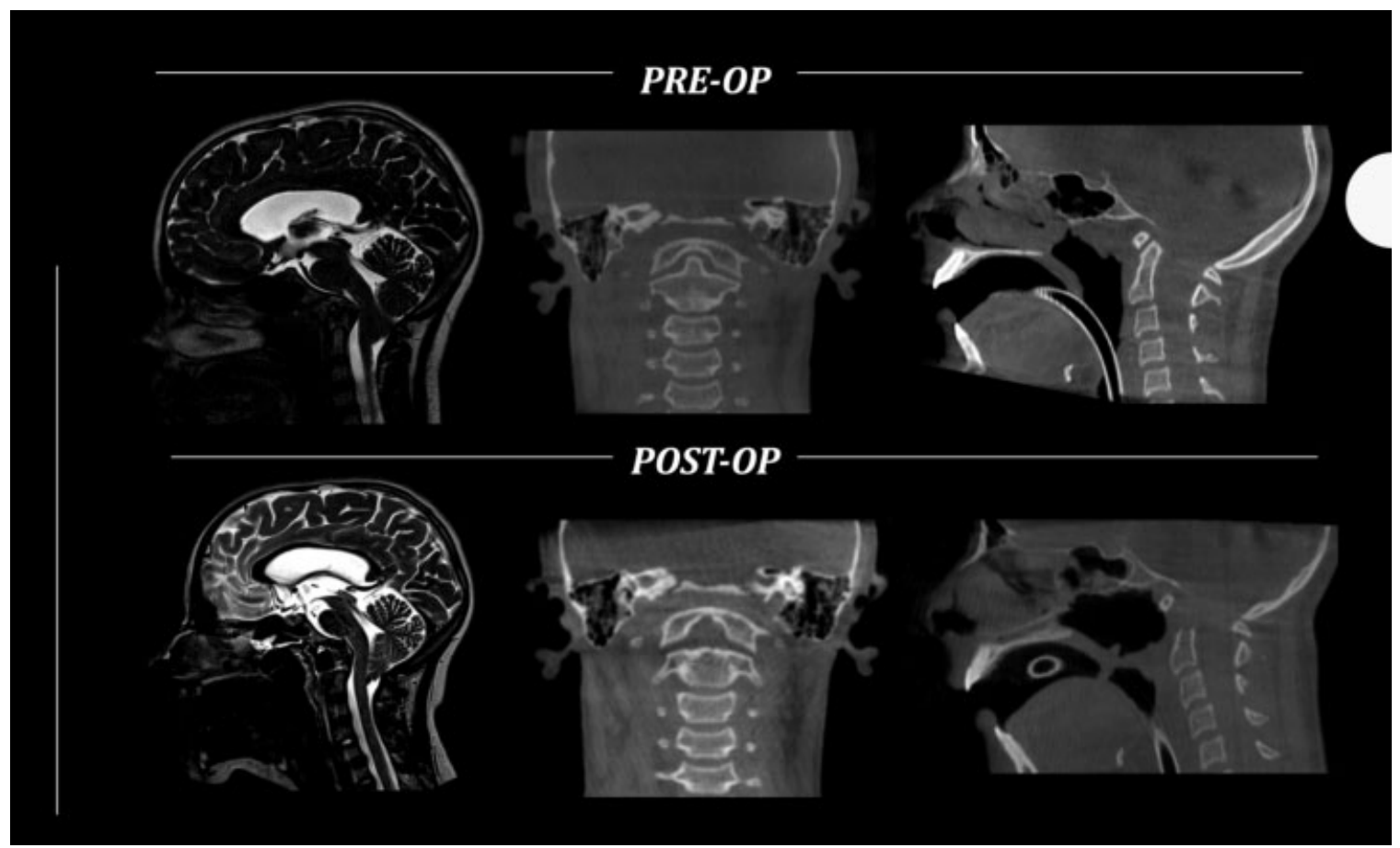

Fig. 1 Pre- and postoperative MRI and CT images. CT, computed tomography; MRI, magnetic resonance imaging.

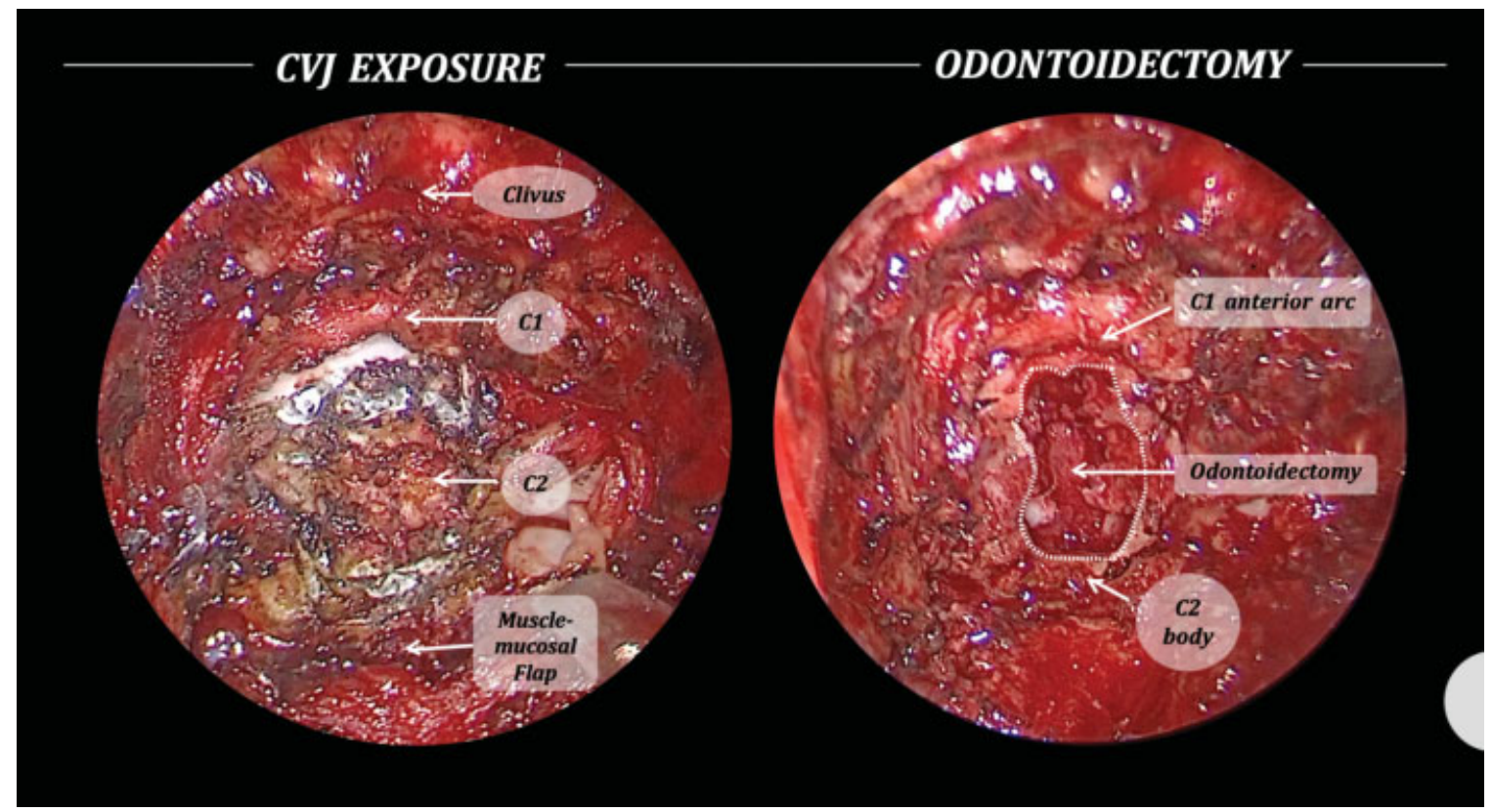

Fig. 2 Intraoperative images of the surgical field before and after the odontoidectomy. CVJ, craniovertebral junction. 